\title{
千岛湖两栖爬行类动物群落结构嵌套分析
}

\author{
王 熙, 王彦平, 丁 平* \\ (浙江大学 生命科学学院, 杭州 310058)
}

摘要: 生境片段化是导致生物多样性降低的主要原因之一。该文采用样线法对千岛湖 23 个岛屿的两栖爬行 类进行了调查，并结合植物种类调查数据和 GIS 技术提取的栖息地景观参数，使用 “BINMATNEST” 软件和 Spearman 相关性分析等方法, 对这些岛屿上的两栖爬行类群落的分布格局及其影响因素进行分析。结果表明, 千 岛湖两栖爬行类群落整体上呈现嵌套分布格局; 嵌套格局与岛屿面积和生境类型相关显著。因此，在制定千岛湖 地区两栖爬行动物保护措施时，应优先考虑面积较大和生境类型多的岛屿。

关键词: 生境片段化; 两栖动物; 爬行动物; 嵌套结构; 保护策略; 千岛湖

中图分类号: Q14; Q915.863; Q915.864 文献标志码：A＼cjkstart文章编号：0254-5853-(2012)05-0439-08

\section{Nested species subsets of amphibians and reptiles in Thousand Island Lake}

\author{
WANG Xi, WANG Yan-Ping, DING Ping* \\ (College of Life Sciences, Zhejiang University, Hangzhou 310058, China)
}

\begin{abstract}
Habitat fragmentation is a main cause for the loss of biological diversity. Combining line-transect methods to survey the amphibians and reptiles on 23 islands on Thousand Island Lake in Zhejiang province, along with survey data on nearby plant species and habitat variables collected by GIS, we used the "BINMATNEST (binary matrix nestedness temperature calculator)" software and the Spearman rank correlation to examine whether amphibians and reptiles followed nested subsets and their influencing factors. The results showed that amphibians and reptiles were significantly nested, and that the island area and habitat type were significantly associated with their nested ranks. Therefore, to effectively protect amphibians and reptiles in the Thousand Islands Lake area we should pay prior attention to islands with larger areas and more habitat types.
\end{abstract}

Key words: Habitat fragmentation; Amphibians; Reptiles; Nestedness; Conservation strategies; Thousand Island Lake

生境片段化是导致生物多样性丧失的主要原 因之一(Burkey, 1995; Vitousek et al, 1997; Laurance et al, 2002; Mac Garigal \& Cushman, 2002)。生境片 段化是指在人为活动和自然干扰下大块连续分布 的自然生境被其他非适宜生境分隔成许多面积较 小的生境斑块的过程。在这个过程中, 不同物种因 对生境片段化敏感程度存在差异而不同程度地占 据这些栖息地斑块, 进而在一定程度上形成嵌套格 局(nestedness) (Zhang et al, 2008)。嵌套格局的概念 最早由 Darlington(1957)提出, 指在一个岛屿生态系 统中, 小岛屿中出现的物种多数也会出现在物种相
对丰富的大岛屿中的分布模式。随后，Patterson \& Atmar (1986)首次系统地提出了在群落水平构建矩 阵进行嵌套分析的方法。从此, 嵌套格局理论在群 落生态学领域被广泛应用于各种栖息地斑块类型 中的群落物种组成和分布格局的研究(Dobson \& Pacala, 1992; Honnay et al, 1999; Fleishman et al, 2002; Greve et al, 2005)。现在，嵌套结构已被证明 是岛屿群落组成的普遍结构。但是由于形成嵌套格 局的机制在不同系统和不同类群中有较大差异 (Wang et al, 2010), 根据已有研究还不能清楚地了 解片段化栖息地中生物群落嵌套格局形成过程的

收稿日期: 2012-02-17; 接受日期: 2012-05-10

基金项目：国家自然科学基金资助项目(31100394, 31170397, 30870315)

*通信作者(Corresponding author), E-mail: dingping@zju.edu.cn 
全貌(Wright et al, 1998)。因此，对于不同栖息地类 型中不同类群的嵌套格局还需要进一步的分析和 研究。

目前已有很多机制用来解释嵌套格局的形成 原因 (Schoener \& Schoener, 1983; Blake, 1991; Baber et al, 2004; Higgins et al, 2006), 主要有 4 种假 说在当前占主导地位(Wright et al, 1998; Chen \& Wang, 2004)：（1）选择性迁移假说(the selective colonization hypothesis): 由于物种扩散能力的差异, 扩散能力强或弱的多数物种都可占领大岛屿; 而扩 散能力强的少数物种占领小岛屿, 从而形成嵌套格 局 (Bird \& Boecklen, 1998; Cook \& Quinn, 1995; Conroy et al, 1999; Loo et al, 2002; Mac Nally et al, 2002)。(2) 选择性灭绝假说(the selective extinction hypothesis): 岛屿面积大小制约物种分布, 要求较大 的最小面积的物种,或具有较小种群的物种, 可能 会从不同面积的岛屿生境中首先灭绝，从而形成嵌 套格局(Patterson \& Atmar, 1986; Bolger et al, 1991; Cutler, 1991; Lomolino, 1996)。（3）生境嵌套假说 ( the habitat nestedneass hypothesis): 物种分布与生 境密切相关, 即嵌套格局是岛屿生境结构呈现嵌套 格局的结果(Blake, 1991; Calmé \& Desrochers, 1999; Honnay et al, 1999; Ficetola \& Bernardi, 2004)。 被动抽样假说(the passive sampling hypothesis): 不 同生境中, 物种多度存在较大差异, 因此在抽取样 本时,多度高的物种被抽中的概率大; 在不同取样 面积下, 数量丰富的物种在小面积中出现的概率和 在大面积的抽样中出现的概率都大, 反之亦然。因此, 不同取样面积的一系列物种组合自然就形成了嵌 套结构(Cutler, 1994; Lomolino, 1996; Andrén, 1994; Nielsen \& Bascompte, 2007)。4 种假说中，除 “被动 抽样” 与物种间多度差异和抽样强度有关(Schouten et al，2007)外，其他三种假说与栖息地特征和物种 生活史特征等因素有关 (Wright et al，1998; Fleishman et al, 2002; Schouten et al, 2007)。

在自然生态系统中，两栖爬行类占有重要地 位。研究表明, 全球两栖爬行类正以超过自然灭绝 的高速率灭绝(Stuart et al, 2004; Whitfield et al, 2007), 这与生境丧失和片段化有直接关系(Chen \& Li, 1990; Pan et al, 2002)。两栖爬行类动物群落结构 的变化, 反映了群落内部关系及群落与其外界环境 的联系。由于两栖动物和爬行动物在不同岛屿之间 的迁移能力较弱(Mac Nally \& Brown, 2001), 物种
交流少, 因此是研究岛屿动物群落组成的良好 类群。

千岛湖独特的自然景观与已形成 50 多年的岛 屿化栖息地为研究栖息地片段化提供了重要实验 平台。本研究以千岛湖人工湖泊岛屿为研究点, 以 两栖爬行类为研究对象, 分析和验证千岛湖岛屿两 栖爬行类群落的分布是否符合嵌套格局，以及面 积、隔离度、植物物种丰富度和生境类型等岛屿景 观参数对嵌套格局的影响。从而探讨影响两栖爬行 类群落分布格局的生态因素，为保护片段化栖息地 内两栖爬行类的生物多样性提供理论依据和策略。

\section{1 研究地概况与研究方法}

\section{1 研究地概况}

千岛湖地处浙江省西部，位于浙江省淳安县境 内 $\left(\mathrm{N} 29^{\circ} 22 \sim 29^{\circ} 50^{\prime}, \mathrm{E} 118^{\circ} 34^{\prime} \sim 11^{\circ} 15^{\prime}\right)$ 。东西长 60 $\mathrm{km}$, 南北宽 $50 \mathrm{~km}$, 景区总面积 $982 \mathrm{~km}^{2}$, 其中湖区 面积 $573 \mathrm{~km}^{2}$ 。千岛湖是 1959 年 9 月建造的新安江 水力发电站，因拦坝蓄水使海拔 $108 \mathrm{~m}$ 以下皆沦为 水域而形成的人工湖水库, 库容量为 $1.784 \times 10^{10} \mathrm{~m}^{3}$, 未被淹没的山峰形成 1078 个面积 $>0.25 \mathrm{hm}^{2}$ 的岛 屿。千岛湖地处亚热带季风气候区的北缘，属亚热 带季风气候区。湖中岛屿植被以天然次生马尾松 (Pinus massoniana) 林为主。年平均气温为 $17{ }^{\circ} \mathrm{C}$, 气 温年较差和日较差小, 年平均降水量为 $1430 \mathrm{~mm}$ (Wang et al, 2009)。

\section{2 岛屿设置}

2009-2011 年夏季，在千岛湖选取 23 个人为 干扰少的岛屿作为取样研究区域(图 1)。所选岛屿面 积从 $<1 \sim>1000 \mathrm{hm}^{2}$, 距陆地最近距离(隔离度)从 640.53 2849.99 m。依据岛屿面积从大到小的顺序 依次标记为 $1 \sim 23$ 号。岛屿方位与景观参数分别见 图 1、表 1。

\section{3 两栖爬行类种类调查}

2009 年、 2010 年和 2011 年的 7-8 月, 采用样 线法调查两栖爬行类。对面积稍大的 1 5 号岛, 采 用“Z”形多条路线，覆盖全岛进行调查; 对 $6 \sim 8$ 号 中型岛，沿岛屿嵴部设置样线，贯通全岛进行调查; 对面积较小的 9 23 号岛, 覆盖全岛面积进行 调查。

调查期间，考虑到两栖爬行类动物的习性，每 天 13:00-17:00 以 $1.5 \mathrm{~km} / \mathrm{h}$ 的速度, 在 $2 \sim 5 \mathrm{~m}$ 范 围内调查蚚蜴类和蛇类动物(Wang et al，2009); 


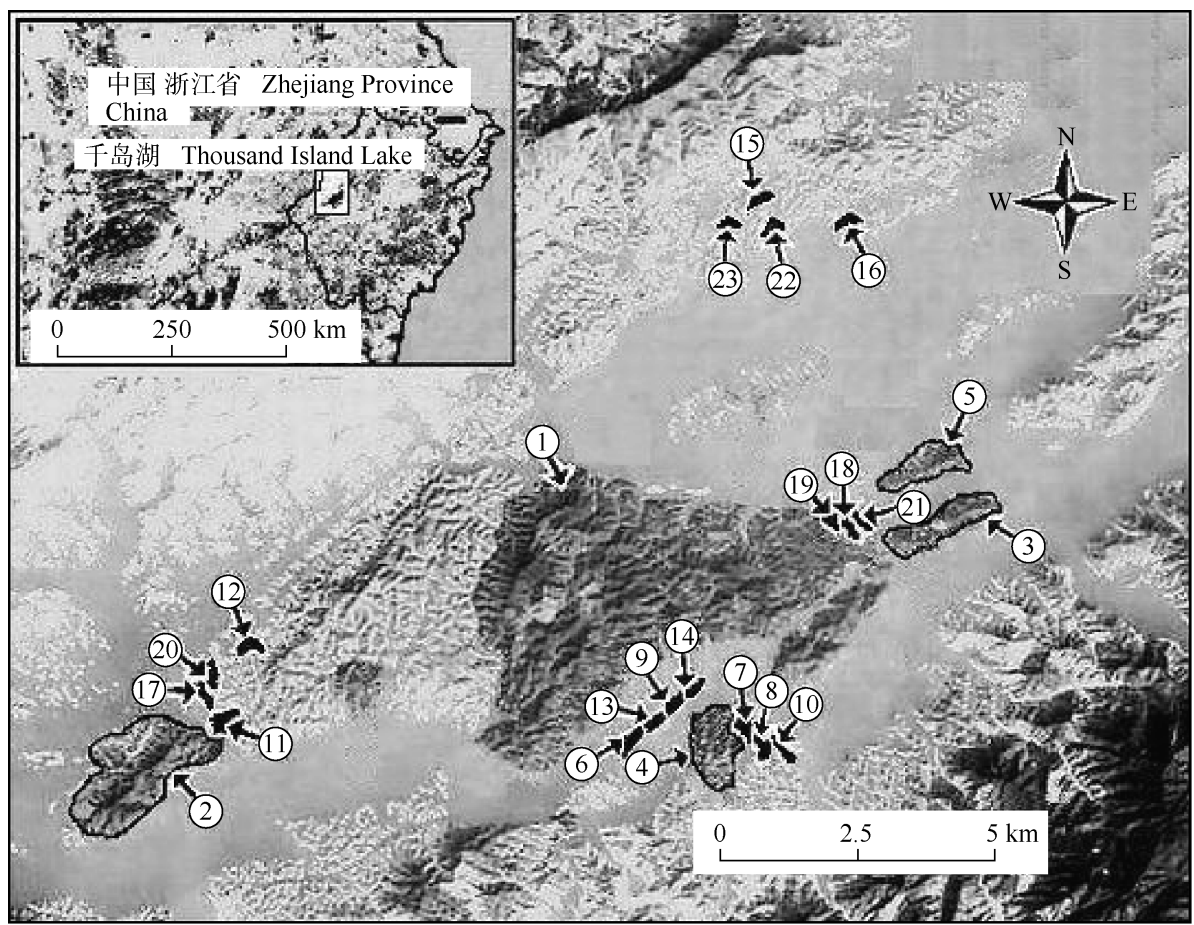

图 1 千岛湖取样岛屿方位图

Fig. 1 Locations of the sampled islands on Thousand Island Lake

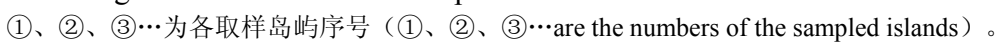

表 1 取样岛屿的景观参数

Tab. 1 Characteristics of 23 islands sampled for the presence of frogs, snakes and lizards in Thousand Island Lake, China

\begin{tabular}{|c|c|c|c|c|c|c|c|}
\hline \multirow{2}{*}{$\begin{array}{l}\text { 岛屿编号 } \\
\text { Island code }\end{array}$} & \multirow{2}{*}{$\begin{array}{c}\text { 岛屿面积 } \\
\text { Island area }\left(\mathrm{hm}^{2}\right)\end{array}$} & \multirow{2}{*}{$\begin{array}{c}\text { 隔离度 } \\
\text { Isolation (m) }\end{array}$} & \multirow{2}{*}{$\begin{array}{c}\text { 生境类型 } \\
\text { Number of habitat } \\
\text { types }(n)\end{array}$} & \multirow{2}{*}{$\begin{array}{c}\text { 植物丰富度 } \\
\text { Plant richness }(n)\end{array}$} & \multicolumn{3}{|c|}{$\begin{array}{c}\text { 嵌套序列 } \\
\text { Nested matrix rank }\end{array}$} \\
\hline & & & & & 蛙类 Frogs & 蛇类 Snakes & 蜥蜴类 Lizards \\
\hline 1 & 1289.23 & 897.41 & 7 & 198 & 1 & 1 & 1 \\
\hline 2 & 143.19 & 1415.09 & 6 & 99 & 2 & 3 & 2 \\
\hline 3 & 55.08 & 953.95 & 5 & 59 & 22 & 2 & 3 \\
\hline 4 & 46.37 & 729.8 & 5 & 51 & 7 & 5 & 5 \\
\hline 5 & 32.29 & 1936.95 & 5 & 57 & 8 & 4 & 4 \\
\hline 6 & 2.9 & 1785.3 & 3 & 85 & 3 & 17 & 9 \\
\hline 7 & 2.83 & 1238.14 & 4 & 86 & 4 & 13 & 17 \\
\hline 8 & 2.29 & 973.85 & 4 & 65 & 11 & 14 & 7 \\
\hline 9 & 1.74 & 2293.25 & 3 & 100 & 6 & 18 & 14 \\
\hline 10 & 1.54 & 711.04 & 3 & 88 & 5 & 10 & 8 \\
\hline 11 & 1.52 & 2849.99 & 3 & 53 & 10 & 6 & 6 \\
\hline 12 & 1.4 & 1760.34 & 3 & 49 & 9 & 11 & 13 \\
\hline 13 & 1.2 & 2128.52 & 3 & 68 & 19 & 7 & 11 \\
\hline 14 & 1.17 & 2453.37 & 3 & 69 & 13 & 20 & 19 \\
\hline 15 & 1.15 & 847.12 & 3 & 33 & 12 & 19 & 20 \\
\hline 16 & 1.03 & 1458.81 & 3 & 36 & 15 & 9 & 18 \\
\hline 17 & 1.01 & 2437.85 & 3 & 29 & 20 & 21 & 22 \\
\hline 18 & 1.01 & 2103.85 & 3 & 36 & 16 & 15 & 12 \\
\hline 19 & 0.86 & 2321.51 & 3 & 56 & 18 & 12 & 15 \\
\hline 20 & 0.83 & 2298.5 & 3 & 50 & 21 & 8 & 16 \\
\hline 21 & 0.8 & 2097.52 & 2 & 80 & 17 & 16 & 21 \\
\hline 22 & 0.67 & 1139.87 & 3 & 39 & 14 & 22 & 10 \\
\hline 23 & 0.59 & 640.53 & 3 & 42 & 23 & 23 & 23 \\
\hline
\end{tabular}

隔离度为岛屿距最近陆地距离; 蛙类、蛇类、蚚蜴类所在的 23 个岛屿的嵌套序列排序为 BINMATNEST 软件最大化排列矩阵的排序, 分别参照表 2 、 表3、表4。

Isolation is the distance between an island and its nearest land; nested matrix ranks of frogs, lizards and snakes on 23 islands are the ranks of maximum permutation matrix generated by the BINMATNEST software. 
18:00-24:00 以 $\sim 0.7 \mathrm{~km} / \mathrm{h}$ 的速度在 LED 强聚光灯 照明下沿着样线搜索前进, 记录距离样线 $2 \sim 3 \mathrm{~m}$ 范围内的蛙类和蛇类(Jaeger，1994)。每个岛屿重复 调查 15 次。

\section{4 栖息地特征参数}

栖息地特征参数包括岛屿面积、隔离度、生境 类型及植物物种丰富度。其中, 隔离度反映两栖爬 行类从陆地迁入岛屿时需要跨越的最小距离(Adler \& Seamon, 1991; Meyer \& Kalko, 2008; Wang et al, 2010，2011)。岛屿面积 $\left(\mathrm{hm}^{2}\right)$ 、隔离度 $(\mathrm{m})$ 均使用 ArcView 3.2 对千岛湖地区景观地形参数图 (1: 10000)进行测量。

2007 年 4-11 月间调查 23 个研究岛屿的生境 类型和植物种类。将所有生境类型划分成针叶林、 阔叶林、针阔混交林、竹林、灌木、杂草和农田 7 个生境类型(Wang et al, 2009)。调查者对岛屿上的 植物种类进行直接辨认, 对辨认不清的植物采样后 对照《浙江种子植物检索鉴定手册》(Zheng, 2005) 和《浙江植物志》(Zhang et al, 1986)进行鉴定。各 岛屿的生境类型和植物物种数(植物物种丰富度值) 详见表 1(Zhang et al, 2008)。

\section{5 嵌套分析方法}

嵌套分析数据是物种在斑块上的出现情况, 分 析前先将数据以矩阵形式排列。其中行代表种类, 列代表地点(即岛屿), 以 “ 1 ” 或 “ 0 ” 表示物种 “出 现” 或 “不出现”。本研究使用 Rodriguez-Girones (2006)提出的 BINMATNEST(binary matrix nestedness temperature calculator)软件基于计算矩阵温度 $\mathrm{T}$ 的 方法来量化嵌套程度。分析时 BINMATNEST 会自 动最大化排列输入的矩阵, 并计算出矩阵温度。比 较实际矩阵温度和使用 Null modal 产生的随机矩阵 (1 000 个)温度, 得到两种矩阵温度差异的显著性。 矩阵比较中应用 BINMATNEST 提供的 null model 3, 该模型根据原矩阵中对应行、列的出现可能性平均 值决定单元格的出现可能性, 在研究中被证明能够 有效控制被动取样对嵌套显著性的影响(RodriguezGirones \& Santamaria, 2006; Moore \& Swihart, 2007)。

序列相关性分析应用 Spearman 来分析嵌套结 构的影响因素。近年来该方法在类似的分析中得到 广泛地使用 (Fernandez-Juricic, 2004; GranadoLoreneioetal, 2007; Schouten et al, 2007)。分析时将 矩阵的行和列依据影响因素(如面积、隔离度等)分
别排序，然后使用 BINMATNEST 最大化排列后的 序列和原始序列进行 Spearman 序列相关性分析。 若相关性显著，说明分析因素对嵌套格局的形成有 显著影响(Chen \& Wang, 2004; Schouten et al, 2007)。各项统计分析计算均在 SPSS13.0 分析软件 中进行。

\section{2 结 果}

\section{$2.1 千$ 岛湖两栖爬行类的嵌套分布格局}

调查共记录蛙类 9 种, 分属 5 科 8 属; 蛇类 11 种，分属 3 科 10 属; 蚚蜴类 5 种，分属 2 科 3 属(表 2-4)。BINMATNEST 软件(null model 3)分析结果表 明: 蛙类、蛇类和蚚蜴类的矩阵温度观测值 $\left(T_{\mathrm{obs}}\right)$ 均 $<$ 矩阵温度预测值 $\left(T_{\text {exp }}\right)(P<0.001)$ (表 5$)$, 说明三 者均表现显著的嵌套格局。

\section{2 千岛湖两栖爬行类嵌套格局的影响因素}

采用 SPSS13.0 统计软件, 以嵌套序列为自变 量, 面积、隔离度等景观参数为因变量(数据见表 1) 进行 Spearman correlation 分析得到如下结果(表 5)。 面积、生境类型和植物丰富度对蛙类嵌套格局的形 成影响显著 $(p<0.05)$, 隔离度的影响不显著; 面积 和生境类型对蛇类嵌套格局的形成影响非常显著 $(P<0.01)$, 植物丰富度和隔离度的影响不显著; 面 积、生境类型和植物丰富度对蚚蜴类嵌套格局形成 的影响非常显著 $(P<0.01)$, 隔离度的影响不显著。

\section{3 讨 论}

\section{1 嵌套格局的影响因素}

岛屿面积大小对千岛湖两栖爬行类群落嵌套 格局的形成有很大影响，这支持选择性灭绝假说。 由于岛屿面积大小在一定程度上反映了动物可用 于生存的资源量和空间范围，通过对具有不同生存 面积需求物种的 “过滤”，群落可能在一定程度上 发生选择性灭绝(Li et al, 1998)。对于需要较大生存 空间范围、生境专属性较高和种群密度较小的物种 而言，灭绝的风险更高(Wang et al, 2010)。因此，这 些物种在较小面积岛屿上出现的几率比在较大面 积岛屿上出现的几率要小。相反, 对于那些对面积 需求较小的物种在小岛和大岛上均有分布，这样便 形成了嵌套格局。此外, 由于岛屿的物种分化程度 往往很低, 尤其是两栖爬行类动物, 所以岛屿动物 种群通常表现出极度发展或极度萎缩的状态，通常 是单一物种数量很大(Halliday \& Adler, 2002)。比如 
表 2 千岛湖蛙类使用 BINMATNEST 软件产生的最大化排列矩阵

Tab. 2 BINMATNEST maximally packed nested matrix for frogs on 23 islands in Thousand Island Lake

\begin{tabular}{|c|c|c|c|c|c|c|c|c|c|c|c|c|c|c|c|c|c|c|c|c|c|c|c|}
\hline \multirow{2}{*}{ 种类 Species } & \multicolumn{23}{|c|}{ 岛屿 Islands } \\
\hline & 1 & 2 & 22 & 7 & 8 & 3 & 4 & 11 & 6 & 5 & 10 & 9 & 19 & 13 & 12 & 15 & 20 & 16 & 18 & 21 & 17 & 14 & 23 \\
\hline $\begin{array}{l}\text { 花臭蛙 } \\
\text { Odorrana schmackeri }\end{array}$ & 1 & 1 & 1 & 1 & 1 & 1 & 1 & 1 & 1 & 1 & 1 & 1 & 1 & 1 & 1 & 1 & 1 & 1 & 1 & 1 & 1 & 1 & 1 \\
\hline $\begin{array}{l}\text { 中华蟾蜍 } \\
\text { Bufo gargarizans gargarizans }\end{array}$ & 1 & 1 & 1 & 0 & 0 & 0 & 0 & 0 & 0 & 0 & 0 & 0 & 0 & 0 & 0 & 0 & 0 & 0 & 0 & 0 & 0 & 0 & 0 \\
\hline $\begin{array}{l}\text { 斑腿泛树蛙 } \\
\text { Polypedates megacephalus }\end{array}$ & 1 & 1 & 0 & 0 & 0 & 0 & 0 & 0 & 0 & 0 & 0 & 0 & 0 & 0 & 0 & 0 & 0 & 0 & 0 & 0 & 0 & 0 & 0 \\
\hline $\begin{array}{l}\text { 饰纹姬娃 } \\
\text { Microhyla ornata }\end{array}$ & 1 & 0 & 0 & 1 & 0 & 0 & 0 & 0 & 0 & 0 & 0 & 0 & 0 & 0 & 0 & 0 & 0 & 0 & 0 & 0 & 0 & 0 & 0 \\
\hline $\begin{array}{l}\text { 中国雨蛙 } \\
\text { Hyla chinensis }\end{array}$ & 1 & 1 & 0 & 0 & 0 & 0 & 0 & 0 & 0 & 0 & 0 & 0 & 0 & 0 & 0 & 0 & 0 & 0 & 0 & 0 & 0 & 0 & 0 \\
\hline $\begin{array}{l}\text { 泽陆蛙 } \\
\text { Fejervarya limnocharis }\end{array}$ & 1 & 1 & 0 & 0 & 0 & 0 & 0 & 0 & 0 & 0 & 0 & 0 & 0 & 0 & 0 & 0 & 0 & 0 & 0 & 0 & 0 & 0 & 0 \\
\hline $\begin{array}{l}\text { 黑斑蛙 } \\
\text { Pelophylax nigromaculata }\end{array}$ & 1 & 0 & 0 & 0 & 0 & 0 & 0 & 0 & 0 & 0 & 0 & 0 & 0 & 0 & 0 & 0 & 0 & 0 & 0 & 0 & 0 & 0 & 0 \\
\hline $\begin{array}{l}\text { 金线蛙 } \\
\text { Pelophylax plancyi plancyi }\end{array}$ & 1 & 0 & 0 & 0 & 0 & 0 & 0 & 0 & 0 & 0 & 0 & 0 & 0 & 0 & 0 & 0 & 0 & 0 & 0 & 0 & 0 & 0 & 0 \\
\hline $\begin{array}{l}\text { 镇海林蛙 } \\
\text { Rana zhenhaiensis }\end{array}$ & 1 & 0 & 0 & 0 & 0 & 0 & 0 & 0 & 0 & 0 & 0 & 0 & 0 & 0 & 0 & 0 & 0 & 0 & 0 & 0 & 0 & 0 & 0 \\
\hline
\end{tabular}

表 3 千岛湖蛇类使用 BINMATNEST 软件产生的最大化排列矩阵

Tab. 3 BINMATNEST maximally packed nested matrix for snakes on 23 islands in Thousand Island Lake

\begin{tabular}{|c|c|c|c|c|c|c|c|c|c|c|c|c|c|c|c|c|c|c|c|c|c|c|c|}
\hline \multirow{2}{*}{ 种类 Species } & \multicolumn{23}{|c|}{ 岛屿 Islands } \\
\hline & 1 & 3 & 2 & 5 & 4 & 17 & 13 & 14 & 18 & 10 & 6 & 11 & 7 & 20 & 19 & 9 & 21 & 15 & 12 & 8 & 16 & 22 & 23 \\
\hline $\begin{array}{l}\text { 王锦蛇 } \\
\text { Elaphe carinata }\end{array}$ & 1 & 1 & 1 & 1 & 1 & 1 & 1 & 1 & 1 & 1 & 1 & 1 & 1 & 1 & 0 & 1 & 1 & 1 & 1 & 1 & 0 & 0 & 0 \\
\hline $\begin{array}{l}\text { 乌梢蛇 } \\
\text { Zaocys dhumnades }\end{array}$ & 1 & 1 & 1 & 0 & 0 & 1 & 0 & 1 & 0 & 0 & 0 & 0 & 0 & 1 & 1 & 0 & 0 & 0 & 0 & 0 & 0 & 0 & 0 \\
\hline $\begin{array}{l}\text { 银环蛇 } \\
\text { Bungarus multicinctus }\end{array}$ & 1 & 1 & 1 & 0 & 1 & 0 & 1 & 0 & 0 & 0 & 0 & 1 & 1 & 0 & 0 & 0 & 0 & 0 & 0 & 0 & 0 & 0 & 0 \\
\hline $\begin{array}{l}\text { 虎斑颈槽蛇 } \\
\text { Rhabdophis tigrinus }\end{array}$ & 1 & 1 & 1 & 1 & 1 & 0 & 0 & 1 & 0 & 0 & 0 & 0 & 0 & 0 & 0 & 0 & 0 & 0 & 0 & 0 & 0 & 0 & 0 \\
\hline $\begin{array}{l}\text { 眼镜蛇 } \\
\text { Naja atra }\end{array}$ & 1 & 0 & 0 & 1 & 0 & 0 & 0 & 0 & 1 & 1 & 0 & 0 & 0 & 0 & 0 & 0 & 0 & 0 & 0 & 0 & 0 & 0 & 0 \\
\hline $\begin{array}{l}\text { 竹叶青 } \\
\text { Trimeresurus stejnegeri stejnegeri }\end{array}$ & 1 & 0 & 1 & 0 & 0 & 0 & 1 & 0 & 0 & 0 & 1 & 0 & 0 & 0 & 0 & 0 & 0 & 0 & 0 & 0 & 0 & 0 & 0 \\
\hline $\begin{array}{l}\text { 尖吻蝮 } \\
\text { Deinagkistrodon acutus }\end{array}$ & 1 & 1 & 0 & 1 & 0 & 1 & 0 & 0 & 0 & 0 & 0 & 0 & 0 & 0 & 0 & 0 & 0 & 0 & 0 & 0 & 0 & 0 & 0 \\
\hline $\begin{array}{l}\text { 翠青蛇 } \\
\text { Cyclophiops major }\end{array}$ & 1 & 1 & 1 & 1 & 1 & 0 & 0 & 0 & 0 & 0 & 0 & 0 & 0 & 0 & 0 & 0 & 0 & 0 & 0 & 0 & 0 & 0 & 0 \\
\hline $\begin{array}{l}\text { 龟壳花蛇 } \\
\text { Trimeresurus mucrosquamatus }\end{array}$ & 1 & 1 & 0 & 0 & 0 & 0 & 0 & 0 & 0 & 0 & 0 & 0 & 0 & 0 & 0 & 0 & 0 & 0 & 0 & 0 & 0 & 0 & 0 \\
\hline $\begin{array}{l}\text { 中国小头蛇 } \\
\text { Oligodon chinensis }\end{array}$ & 1 & 1 & 0 & 0 & 0 & 0 & 0 & 0 & 0 & 0 & 0 & 0 & 0 & 0 & 0 & 0 & 0 & 0 & 0 & 0 & 0 & 0 & 0 \\
\hline $\begin{array}{l}\text { 滑鼠蛇 } \\
\text { Ptyas mucosus }\end{array}$ & 1 & 0 & 0 & 0 & 0 & 0 & 0 & 0 & 0 & 0 & 0 & 0 & 0 & 0 & 0 & 0 & 0 & 0 & 0 & 0 & 0 & 0 & 0 \\
\hline
\end{tabular}

表 4 千岛湖蜥蜴类使用 BINMATNEST 软件产生的最大化排列矩阵

Tab. 4 BINMATNEST maximally packed nested matrix for lizards on the studied islands in Thousand Island Lake

\begin{tabular}{|c|c|c|c|c|c|c|c|c|c|c|c|c|c|c|c|c|c|c|c|c|c|c|c|}
\hline \multirow{2}{*}{ 种类 Species } & \multicolumn{23}{|c|}{ 岛屿 Islands } \\
\hline & 1 & 2 & 3 & 5 & 4 & 9 & 17 & 7 & 14 & 8 & 6 & 13 & 11 & 19 & 20 & 18 & 22 & 12 & 15 & 16 & 21 & 10 & 23 \\
\hline 中国石龙子 Eumeces chinensis & 1 & 1 & 1 & 1 & 1 & 1 & 0 & 1 & 1 & 1 & 1 & 1 & 0 & 0 & 0 & 0 & 1 & 1 & 1 & 1 & 1 & 1 & 0 \\
\hline 北草蜊 Takydromus septentrionalis & 1 & 1 & 1 & 1 & 1 & 1 & 0 & 0 & 0 & 0 & 1 & 1 & 1 & 1 & 1 & 1 & 0 & 0 & 0 & 0 & 0 & 0 & 0 \\
\hline 蓝尾石龙子 Eumeces elegans & 1 & 1 & 0 & 0 & 1 & 0 & 0 & 1 & 1 & 1 & 0 & 0 & 0 & 0 & 0 & 0 & 0 & 0 & 0 & 0 & 0 & 0 & 0 \\
\hline 宁波滑蜘 Scincella modestum & 1 & 0 & 1 & 1 & 0 & 0 & 1 & 0 & 0 & 0 & 0 & 0 & 0 & 0 & 0 & 0 & 0 & 0 & 0 & 0 & 0 & 0 & 0 \\
\hline 印度蜓蜥 Sphenormorphus indicus & 1 & 1 & 0 & 0 & 0 & 0 & 0 & 0 & 0 & 0 & 0 & 0 & 0 & 0 & 0 & 0 & 0 & 0 & 0 & 0 & 0 & 0 & 0 \\
\hline
\end{tabular}


表 5 千岛湖 23 个岛屿两栖爬行类嵌套分析结果，表中给出矩阵温度观测值 $\left(T_{\mathrm{obs}}\right)$,

矩阵温度预测值 $\left(\boldsymbol{T}_{\text {exp }}\right)$ 和使用零模型 3 得到的随机检验 $\boldsymbol{P}$ 值

Tab. 5 Results of the nested analyses for all amphibians and reptiles on the 23 islands in Thousand Island Lake; showing observed matrix temperatures $\left(T_{\text {obs}}\right)$, expected nested temperatures $\left(T_{\text {exp }}\right)$, and Monte Carlo-derived probabilities that the matrix was randomly generated under null model 3

\begin{tabular}{|c|c|c|c|c|c|c|c|}
\hline & \multirow{2}{*}{$\begin{array}{c}\text { 矩阵温度观测值 } \\
T_{\mathrm{obs}}\end{array}$} & \multirow{2}{*}{$\begin{array}{c}\text { 矩阵温度预测值 } \\
T_{\exp }\end{array}$} & \multirow{2}{*}{$\begin{array}{c}\text { 显著水平 } \\
P\end{array}$} & \multicolumn{4}{|c|}{$\begin{array}{c}\text { 与岛屿特征的秩相关 } \\
\text { Rank correlation with island }\end{array}$} \\
\hline & & & & 面积 Area & 隔离度 DNM & 植物丰富度 PSR & 生境类型 HT \\
\hline 蛙类 NR & 0.23 & 11.62 & $<0.001$ & $-0.709^{* *}$ & 0.335 & $-0.487^{*}$ & $-0.720 * *$ \\
\hline 蛇类 NR & 4.13 & 23.46 & $<0.001$ & $-0.654 * *$ & -0.083 & -0.381 & $-0.594 * *$ \\
\hline 蜊蜴类 NR & 8.33 & 29.35 & $<0.001$ & $-0.767 * *$ & -0.105 & $-0.443^{*}$ & $-0.781 * *$ \\
\hline
\end{tabular}

NR: 嵌套序列(Nestedness ranking); DNM: 距最近陆地距离(Distance to the nearest mainland); PSR: 植物丰富度(Plant species richness); HT: 生境类型 (Habitat type)。*P<0.05, ** $P<0.01$ (Spearman rank correlations).

在本研究中花臭蛙在所有调查岛上均有分布, 而且 数量众多。

岛屿的生境类型对千岛湖两栖爬行类群落嵌 套格局的形成也有很大影响, 这符合生境嵌套结构 假说。Wang et al $(2009,2010)$ 的研究表明, 蚚蜴类对 生境片段化十分敏感，同时生境异质性对蚚蜴类的 嵌套格局有显著影响。但是多数物种偏爱某一类型 的生境，而生境类型在岛屿间的分布并不是随机的， 而是呈现一种嵌套的分布，生境的嵌套分布最终将 导致物种分布的嵌套结构 (Simberloff \& Martin, 1991; Calmé \& Desrochers, 1999)。千岛湖岛屿生境 嵌套的形成，可能是由岛屿间不同的土壤类型和水 文地理学因素造成的(Wang et al, 2010)。

植物物种丰富度对蛙类和蚚蜴类的嵌套格局 也有显著影响, 这可能是由于植物物种丰富度高的 栖息地能为蛙类和蚚蜴类提供繁殖和掩蔽场所 (Simberloff \& Martin, 1991; Calmé \& Desrochers, 1999)。这些掩蔽场所不仅能让蛙类和蚚蜴类躲避天 敌捕食, 也能减少蛙类因阳光照射而造成的水分散 失，这是由蛙类的皮肤通透性特点造成的; 而对于 蛇类, 其皮肤具有的鳞片能有效防止水分散失, 这 可能是蛇类的嵌套格局与植物物种丰富度关系不 明显的原因之一(Halliday \& Adler, 2002)。因此, 由 岛屿间植被丰富度差异造成的不同程度的限制作 用可能使蛙类和蚚蜴类在一定程度上呈现出嵌套 格局，而对蛇类影响不大。

隔离度不影响两栖爬行类群落嵌套格局的形 成，不支持选择性迁移假说。根据该假说，由于物 种扩散能力的差异, 扩散能力强的物种将占领多数 岛屿, 而扩散能力差的物种往往只能在隔离度较 小、面积较大的岛屿定居(Cook \& Quinn, 1995)。这
样，大岛屿自然拥有物种库中的多数物种，而小岛 屿只拥有少于扩散能力强的物种, 从而形成嵌套结 构(Chen \& Wang, 2004)。由于两栖爬行类动物迁移 能力较弱, 迁移距离有限, 可能在千岛湖岛屿形成 后很少在岛屿间发生迁移(Wang et al, 2010)。因此不 能用选择性迁入假说来解释两栖爬行类群落嵌套 格局的形成。

尽管嵌套格局广泛存在于许多研究类群 (Wright et al, 1998), 但迄今为止两栖爬行类还很少 被关注。目前仅有的少数研究表明, 片段化生境的 面积大小是决定两栖爬行类嵌套格局的主要因素 (Li et al, 1998; Mac Nally \& Brown, 2001; Hecnar et al, 2002), 这与我们的研究结果相似。但是, Watling et al (2009) 对新热带片段化森林岛屿(forest islands) 两栖爬行类的研究却发现, 隔离度是影响嵌套格局 形成的主要因素, 这与我们的研究结果相反。隔离 度在本研究和 Watling et al (2009) 研究中作用的不 同，可能主要是由于二者之间在基质(matrix)类型、 隔离时间、隔离度变化程度等方面的不同所产生 的。但是, 关于生境类型和植物物种丰富度在两栖 爬行类群落嵌套格局形成中的作用, 除本研究外, 其余研究未有涉及，需深入研究。

\section{2 保护建议}

嵌套结构在保护生物学上具有特殊意义, 为自 然保护区的最优化设计提供了新的视角(Chen \& Wang, 2004)。我们通过对千岛湖两栖爬行类群落的 嵌套程度及其影响因素进行研究, 发现两栖爬行类 群落整体上呈现嵌套分布格局, 且又与岛屿面积和 生境类型显著相关。因此, 我们认为千岛湖两栖爬 行类动物群落嵌套格局的形成是由选择性灭绝和 生境嵌套共同决定的。研究表明，那些面积较大、 
植物物种丰富度高和生境类型多的岛屿包含更多 的物种, 能够为两栖爬行类提供良好的生存和栖息 环境(Wang et al, 2009)。因此, 我们建议在制定千岛 湖两栖爬行类物种多样性保护对策时，应该对那些 面积较大、植物物种丰富度高和生境类型多的岛屿 予以重点关注。

\section{3 结论与展望}

嵌套格局是研究群落结构的有效工具(Worthen, 1996)。首先, 嵌套格局不仅关注群落的物种丰度 (species richness)和多度(abundance), 更重要的是它 还关注群落组成(community composition)(Worthen,

\section{参考文献:}

Adler GH, Seamon JO. 1991. Distribution and abundance of a tropical Rodent, on islands in Panama [J]. J Trop Ecol, 7(3): 349-360.

Andrén H. 1994. Can one use nested subset pattern to reject the random sample hypothesis? Examples from boreal bird communities [J]. Oikos, 70(3): 489-491.

Baber MJ, Fleishman E, Babbitt KJ, Tarr TL. 2004. The relationship between wetland hydroperiod and nestedness patterns in assemblages of larval amphibians and predatory macroinvertebrates [J]. Oikos, 107: 16-27.

Bird BM, Boecklen WJ. 1998. Nestedness and migratory status of avian assemblages in North America and Europe [J]. Biodivers Conserv, 7(10): 1325-1331.

Blake JG. 1991. Nested subsets and the distribution of birds on isolated woodlots [J]. Oecologia, 5(1): 58-66.

Bolger DT, Alberts AC, Soule ME. 1991. Occurrence patterns of bird species in habitat fragments: sampling, extinction, and nested species subsets [J]. Amer Naturalist, 137(2): 155-166.

Burkey TV. 1995. Extinction rates in archipelagoes: implications for populations in fragmented habitats [J] . Conserv Biol, 9(3): 527-541.

Calmé S, Desrochers A. 1999. Nested bird and micro-habitat assemblages in a peatland archipelago [J]. Oecologia, 118(3): 361-370.

Chen HJ, Li FL. 1990. The effect of human action on community structure in amphibia [J]. Zool Res, 11(3): 199-203. [陈火结, 李芳林. 1990. 人 类活动对两栖动物群落结构的影响. 动物学研究, 11(3): 199-203.]

Chen SH, Wang YJ. 2004. Nestedness pattern of insular community assemblages and its application [J]. Chn J Ecol, 23(3): 81-87. [陈水华, 王玉军. 2004. 岛屿群落组成的嵌套格局及其应用. 生态学杂志, 23(3): 81-87.]

Conroy CJ, Demboski JR, Cook JA. 1999. Mammalian biogeography of the Alexander Archipelago of Alaska: a north temperate nested fauna [J]. $J$ Biogeogr, 26(2): 343-352.

Cook RR, Quinn JF. 1995. The influence of colonization in nested species subsets [J]. Oecologia, 102(4): 413-424.

Cutler A. 1991. Nested faunas and extinction in fragmented habitats [J]. Conserv Biol, 5(4): 496-505.

Cutler AH. 1994. Nested biotas and biological conservation: metrics, mechanisms and meaning of nestedness [J]. Landscape Urban Plan, 28(1): 73-82.

Darlington PJ. 1957. Zoography: the Geographical Distribution of Animals [M]. New York: John Wiley.

Dobson AP, Pacala SW. 1992. The parasites of Anolis lizards the northern
1996); 其次，嵌套格局分析仅仅基于物种是否出现 的 “存在” (presence)与 “不存在” (absence) 数据。 由于该类数据非常容易获得，因此嵌套格局为分析 群落结构提供了经济有效的研究工具(Wright et al, 1998)。鉴于目前两栖爬行类正经历全球性的种群下 降和灭绝危机(Stuart et al, 2004; Whitfield et al, 2007), 而现在有关该类群嵌套格局研究还很少被 关注，因此以后应该加强该领域的研究。

致谢: 千岛湖本地的章庙元等渔民为野外工作 提供了大量人力帮助，特此致谢!

Lesser Antilles II. The structure of the parasite community $[\mathrm{J}]$. Oecologia, 91(1): 118-125.

Fernandez-Juricic E. 2004. Spatial and temporal analysis of the distribution of forest specialists in an urban-fragmented landscape (Madrid, Spain): implications for local and regional bird conservation [J]. Landscape Urban Plan, 69(1): 17-32.

Ficetola GF, Bernardi FD. 2004. Amphibians in a human-dominated landscape: the community structure is related to habitat features and isolation [J]. Biol Conserv, 119(2): 219-230.

Fleishman E, Betrus CJ, Blair RB, Nally RM, Murphy DD. 2002. Nestedness analysis and conservation planning: the importance of place, environment, and life history across taxonomic groups $[\mathrm{J}]$. Oecologia, 133(1): 78-89.

Granado-Lorencio C, Cerviá J, Lima C. 2007. Floodplain lake fish assemblages in the Amazon River: directions in conservation biology [J]. Biodivers Conserv, 16(3): 679-692.

Greve M, Gremmen NM, Gaston KJ, Chown SL. 2005. Nestedness of Southern Ocean Island biotas: ecological perspectives on a biogeographical conundrum [J]. J Biogeogr, 32(1): 155-168.

Halliday T, Adler K. 2002. The New Encyclopedia of Reptiles and Amphibians [M]. Oxford: Oxford University Press.

Hecnar SJ, Casper GS, Russell RW, Hecnar DR, Robinson JN. 2002. Nested species assemblages of amphibians and reptiles on islands in the Laurentian Great Lakes [J]. J Biogeogr, 29(4): 475-489.

Higgins CL, Willig MR, Strauss RE. 2006. The role of stochastic processes in producing nested patterns of species distribution [J]. Oikos, 114(1): 159-167.

Honnay O, Henry M, Choppin P. 1999. Nested plant communities in deciduous forest fragments: species relaxation or nested habitats $[\mathrm{J}]$. Oikos, 84(1): 119-129.

Jaeger RG. 1994. Transect Sampling. Measuring and Monitoring Biological Diversity: Standard Methods for Amphibians [M]. Washington: Smithsonian Institution Press.

Laurance WF, Lovejoy TE, Vasconcelos HL, Bruna E M, Didham R K, Stouffer PC, Gascon C, Bierregaard RO, Laurance SG, Sampaio E. 2002. Ecosystem decay of Amazonian forest fragments: a 22-year investigation [J]. Convers Biol, 16(3): 605-618.

Li YM, Niemelä J, Li DM. 1998. Nested distribution of amphibians in the Zhoushan archipelago, China: can selective extinction cause nested subsets of species? [J]. Oecologia, 113(4): 557-564.

Lomolino MV. 1996. Investigating causality of nestedness of insular 
communities: selective migrations or extinctions [J]? J Biogeogr, 23(5): 699-703.

Loo SE, Mac Nally R, Quinn GP. 2002. An experimental examination of colonization as a generator of biotic nestedness [J]. Oecologia, 132(1): 118-124.

McGarigal K, Cushman SA. 2002. Comparative evaluation of experimental approaches to the study of habitat fragmentation effects [J]. Ecol Appl, 12(2): 335-345.

Mac Nally R, Brown GW. 2001. Reptiles and habitat fragmentation in the box-ironbark forests of central Victoria, Australia: predictions, compositional change and faunal nestedness [J]. Oecologia, 128(1): 116-125.

Mac Nally R, Horrocks G, Bennett AF. 2002. Nestedness in fragmented landscapes: birds of the box-ironbark forests of south-eastern Australia [J]. Ecography, 25(6): 651-660.

Meyer CFJ, Kalko EKV. 2008. Bat assemblages on Neotropical land-bridge islands: nested subsets and null model analyses of species co-occurrence patterns [J]. Divers Distrib, 14(4): 644-654

Moore JE, Swihart RK. 2007. Toward ecologically explicit null models of nestedness [J]. Oecologia, 152(4): 763-777.

Nielsen A, Bascompte J. 2007. Ecological networks, nestedness and sampling effort [J]. J Ecol, 95(5): 1134-1141.

Pan XF, Zhou W, Zhou YW, Jiang GS. 2002. A summary of studies on amphibian population ecology in China [J]. Zool Res, 23(5): 426-436. [潘晓赋, 周伟, 周用武, 江桂盛. 2002. 中国两栖类种群生态研究 概述. 动物学研究, 23(5): 426-436.]

Patterson BD, Atmar W. 1986. Nested subsets and the structure of insular mammalian faunas and archipelagos [J]. Biol J Linn Soc, 28(1-2): 65-82.

Rodriguez-Girones MA, Santamaria L. 2006. A new algorithm to calculate the nestedness temperature of presence-absence matrices [J]. $J$ Biogeogr, 33(5): 924-935.

Schoener TW, Schoener A. 1983. Distribution of vertebrates on some very small islands. I. Occurrence sequences of individual species [J]. J Anim Ecol, 52(1): 209-235.

Schouten MA, Verweij PA, Barendregt A, Kleukers RJM, de Ruiter PC. 2007. Nested assemblages of Orthoptera species in the Netherlands: the importance of habitat features and life-history traits [J]. J Biogeogr, 34(11): 1938-1946.
Simberloff D, Martin JL. 1991. Nestedness of insular avifaunas: simple summary statistics masking complex species patterns [J]. Ornis Fennica, 68: 178-192.

Stuart SN, Chanson JS, Cox NA, Young BE, Rodrigues ASL, Fischman DL, Waller RW. 2004. Status and trends of amphibians declines and extinctions worldwide [J]. Science, 306(5702): 1783-1786.

Vitousek P, Mooney HA, Lubchenco J, Melillo JM. 1997. Human domination of Earth's ecosystems [J]. Science, 277(5325): 494-499.

Watling JI, Gerow K, Donnelly MA. 2009. Nested species subsets of amphibians and reptiles on Neotropical forest islands [J]. Anim Conserv, 12(5): 467-476.

Wang YP, Zhang J, Feeley KJ, Jiang PP, Ding P. 2009. Life-history traits associated with fragmentation vulnerability of lizards in the Thousand Island Lake, China [J]. Anim Conserv, 12(4): 329-337.

Wang YP, Bao YX, Yu MJ, Xu GF, Ding P. 2010. Nestedness for different reasons: the distributions of birds, lizards and small mammals on islands of an Inundated Lake [J]. .Divers Distrib, 16(5): 862-873.

Wang YP, Chen, SH Ding P. 2011. Testing multiple assembly rule models in avian communities on islands of an inundated lake, Zhejiang Province, China [J]. J Biogeogr, 38(7): 1330-1344.

Whitfield SM, Bell KE, Philippi T, Sasa M, Bolaños F, Chaves G, Savage JM, Donnelly MA. 2007. Amphibian and reptile declines over 35 years at La Selva, Costa Rica [J]. Proc Natl Acad Sci USA, 104(20): 8352-8356.

Worthen WB. 1996. Community composition and nested subset analyses: basic descriptors for community ecology [J]. Oikos, 76(3): 417-426.

Wright DH, Patterson BD, Mikkelson GM, Cutler A, Atmar W. 1998. A comparative analysis of nested subset patterns of species composition [J]. Oecologia, 113(1): 1-20.

Zhang JC, Wang YP, Jiang PP, Li P, Yu MJ, Ding P. 2008. Nested analysis of passeriform bird assemblages in the Thousand Island Lake region [J]. Biodiver Sci, 16(4): 321-331. [张竞成, 王彦平, 蒋萍萍, 李鹏, 于明 坚, 丁平. 2008. 千岛湖雀形目鸟类群落嵌套结构分析. 生物多样性, 16(4): 321-331.]

Zhang SY, Ding BY. 1986. Flora of Zhejiang [M]. Hangzhou: Zhejiang Science and Technology Press. [章绍尧, 丁炳扬. 1986. 杭州: 浙江 植物志. 浙江科学与技术出版社.]

Zheng CZ. 2005. Keys of Seed Plants in Zhejiang [M]. Hangzhou: Zhejiang Science and Technology Press. [郑朝宗. 2005. 浙江种子植物检索鉴 定手册. 杭州: 浙江科学与技术出版社.] 